\title{
Value-added Utilization of Caustic Soda Lye from Cold Caustic Extraction Process in the Pulp Mill
}

\begin{abstract}
Chen Gong, Jian-Ping Ni, Shu-Jie Fan, Yu Zhang, Bin Yang, Zhen-Hua Su, ${ }^{*}$ and Chao Tian*

Three approaches were examined for the reutilization of cold caustic extraction (CCE) lye. The term CCE lye refers here to the used $\mathrm{NaOH}$ solution with relatively high alkali content, which could be wasted if not reused properly. The results showed that CCE lye can be effectively applied in deinking, alkaline hydrogen peroxide bleaching, and oxygen delignification processes. The CCE lye improved the physical strength of the dried sheet of pulp, as the tensile index increased by $17.9 \%$ to $40.5 \%$, the tear index increased by $2.9 \%$ to $22.2 \%$, and the burst index increased by $10.5 \%$ to $41.0 \%$. The results demonstrated the feasibility of integrating the CCE process of dissolving pulp production with regular pulping and bleaching processes.
\end{abstract}

Keywords: CCE lye; Deinking; Hydrogen peroxide bleaching; Oxygen delignification

Contact information: China National Pulp and Paper Research Institute, P. O. Box 100102, Beijing, China; National Engineering Lab for Pulp and Paper, P. O. Box 100102, Beijing, China;

*Corresponding authors: ChaoTian: hjbhjs@vip.126.com; Zhen-Hua Su: suzhh0722@126.com

\section{INTRODUCTION}

Dissolving-grade pulps have been widely used for the production of regenerated cellulose fibers and cellulose derivatives. Dissolving pulp is a purified bleached pulp with high cellulose content (90\% to 99\%) and low contents of hemicelluloses, lignin, and extractives (Sixta 2006). The dissolving pulp market has flourished over the last decade. In 2019, global production of dissolving pulp reached 7.5 million tons, with a year-on-year increase of $6.1 \%$ compared to 2018 . In China, the supply of dissolving pulp has always been less than demand. From 2015 to 2019, the production capacity of dissolving pulp increased from 1.22 million tons to 1.58 million tons, while the import volume also increased from 2.25 million tons to 3.06 million tons (China Paper Association 2020). Therefore, it is necessary to develop high-quality and low-cost dissolving pulp to alleviate the difference between supply and demand. One promising approach is applying cold caustic extraction (CCE) to upgrade a paper-grade pulp to a dissolving-grade pulp (Tian et al. 2016; Gong et al. 2017).

Cold caustic extraction can effectively upgrade pre-hydrolysis kraft pulp to acetategrade dissolving pulp at $20{ }^{\circ} \mathrm{C}$ to $50{ }^{\circ} \mathrm{C}$ in $10 \%$ to $20 \%$ alkaline solution, aiming to selectively dissolve short chain carbohydrates, mainly hemicelluloses (Puls et al. 2006; Sixta et al. 2013; Arnoul-Jarriault et al. 2014). However, there may be some difficulties in practical application of CCE due to the recovery of the relatively high concentration of alkaline solution and the presence of hemicelluloses in the CCE filtrate.

In a previous study, a sequential extraction process was applied to isolate the hemicelluloses from the CCE filtrate for value-added products, while the caustic liquor can be reused for a subsequent CCE process (Gong et al. 2017). This study further explored 
the feasibility of reutilization of CCE lye for its alkalinity and rich hemicelluloses content. Studies have been conducted on the properties of recycled pulp when adding hemicelluloses. Results indicate that hemicelluloses can be effectively applied to improve the strength properties of recycled pulp. The recycled paper tensile strength and burst strength can be improved by $30.0 \%$ and $44.1 \%$, respectively, with addition of $2 \%$ polyxylose, due to the improved fiber bonding (Dong et al. 2009). According to Cao et al. (1998) and Oksanen et al. (1997), there is a linear relationship between the physical properties of recycled paper and xylose content in pulp. Zhang et al. (2002) also reported that the paper strength was effectively improved when adding hemicelluloses to the pulp.

Although alkaline solution is used in bleaching, oxygen delignification, and deinking processes in the traditional pulp and papermaking industry, there has been a lack of results of CCE lye reutilization reported in the literature. This study explored the feasibility of applying used CCE lye in the above processes.

\section{EXPERIMENTAL}

\section{Materials}

The bleached softwood kraft pulp (for CCE process) and old newspaper (ONP) (for deinking process) were provided by a manufacturer in China. The unbleached softwood kraft pulp was prepared in the laboratory for oxygen bleaching and hydrogen peroxide bleaching. The chemicals used were of analytical grade, purchased from Aladdin Chemicals Co. Ltd. (Shanghai, China).

\section{CCE Lye}

The CCE experiments were performed with a pulp consistency of $10 \%$, an alkali concentration of $15 \%$, and a temperature of $30{ }^{\circ} \mathrm{C}$. The CCE treatment of the bleached softwood kraft pulp was performed in polyethylene bags heated in a water bath for $60 \mathrm{~min}$. The bags were taken out every 15 min for mixing by kneading manually for $10 \mathrm{~s}$ to $15 \mathrm{~s}$. After the CCE treatment, the pulp samples were filtered, and the filtrate was collected as CCE lye.

\section{Hemicelluloses Recovery and Characterization}

The two-step extraction process (35\% ethanol, then $50 \%$ ethanol) was applied to isolate the hemicelluloses from the CCE lye. The precipitated fraction was separated from the liquid phase by centrifugation, washed with $95 \%$ ethanol, vacuum dried at $60{ }^{\circ} \mathrm{C}$, and collected for analysis.

\section{Reutilization of the CCE Lye}

To validate the efficiency of using CCE lye as an alkaline source, the CCE lye was applied to replace the $\mathrm{NaOH}$ in the following three processes: deinking, hydrogen peroxide bleaching, and oxygen delignification. Three conditions were performed, as follows: (A) control, using $\mathrm{NaOH}$ as alkaline source; (B) using $\mathrm{CCE}$ lye to replace $\mathrm{NaOH}$; and (C) using CCE lye plus $0.5 \%$ isolated hemicelluloses (based on raw material) to replace $\mathrm{NaOH}$. The experimental conditions of deinking, hydrogen peroxide bleaching, and oxygen delignification are summarized in Table 1. 
Table 1. Experimental Conditions (Chen 1990)

\begin{tabular}{|c|c|c|c|c|c|}
\hline \multicolumn{2}{|c|}{ Deinking } & \multicolumn{2}{c|}{$\mathrm{H}_{2} \mathrm{O}_{2}$ Bleaching } & \multicolumn{2}{c|}{ Oxygen Delignification } \\
\hline $\mathrm{NaOH}(\%)$ & 1.5 & Consistency (\%) & 15 & $\begin{array}{c}\text { Consistency } \\
(\%)\end{array}$ & 10 \\
\hline $\mathrm{Na}_{2} \mathrm{SiO}_{3}(\%)$ & 5 & $\mathrm{NaOH}(\%)$ & 4 & $\mathrm{NaOH}(\%)$ & 2 \\
\hline $\mathrm{H}_{2} \mathrm{O}_{2}(\%)$ & 2 & $\mathrm{H}_{2} \mathrm{O}_{2}(\%)$ & 6 & Temperature $\left({ }^{\circ} \mathrm{C}\right)$ & 100 \\
\hline Surfactant (\%) & 1 & $\mathrm{Na}_{2} \mathrm{SiO}_{3}(\%)$ & 2 & Time (min) & 60 \\
\hline Disintegration (min) & 20 & $\mathrm{MgSO}_{4}(\%)$ & 0.05 & $\mathrm{O}_{2}$ pressure (bar) & 5 \\
\hline Flotation (min) & 10 & EDTA (\%) & 0.2 & $\mathrm{pH}$ & $10-12$ \\
\hline & & Temperature $\left({ }^{\circ} \mathrm{C}\right)$ & 85 & Delignification (\%) & $\sim 50$ \\
\hline & & Time (min) & 120 & & \\
\hline
\end{tabular}

\section{Analytical Methods}

Carbohydrate analysis

The constituent neutral sugar and uronic acids in the CCE lye and recovered hemicelluloses were determined by high-performance anion-exchange chromatography (HPAEC) (Peng et al. 2010). The neutral sugars and uronic acids in the CCE lye and recovered hemicelluloses were neutralized and liberated by hydrolysis with $72 \% \mathrm{H}_{2} \mathrm{SO}_{4}$ for $45 \mathrm{~min}$ at $25^{\circ} \mathrm{C}$, followed by high-temperature hydrolysis at $105^{\circ} \mathrm{C}$ for $2.5 \mathrm{~h}$ by dilution to $3 \% \mathrm{H}_{2} \mathrm{SO}_{4}$. After hydrolysis, the samples were diluted and injected into the HPAEC system (Dionex ICS-3000, USA) with an amperometric detector, a CarboPacTMPA-20 column (4 $\mathrm{mm} \times 250 \mathrm{~mm}$, Dionex, Waltham, USA), and a guard PA-20 column ( $3 \mathrm{~mm} \times$ $30 \mathrm{~mm}$, Dionex, Waltham, MA, USA) (column temperature 4 to $60{ }^{\circ} \mathrm{C}$, recommend at 30 ${ }^{\circ} \mathrm{C}$ ). Neutral sugars and uronic acids were separated in isocratic 5-mM NaOH (carbonate free and purged with nitrogen) for $20 \mathrm{~min}$, followed by a $0.75-\mathrm{mM} \mathrm{NaAc}\left(\mathrm{CH}_{3} \mathrm{COONa}\right)$ gradient in $5-\mathrm{mM} \mathrm{NaOH}$ for $15 \mathrm{~min}$ with a flow rate of $0.4 \mathrm{~mL} / \mathrm{min}$. Calibration was performed with standard solutions of L-arabinose, D-glucose, D-xylose, D-mannose, Dgalactose, glucuronic acid, and galacturonic acid.

Handsheets $\left(60 \mathrm{~g} / \mathrm{m}^{2}\right)$ were made and dried in a rapid sheet former (RK-3A, PTI, Vorchdorf, Austria). Brightness and physical strength values of the handsheets were analyzed according to ISO 2470 (2016), T494 om-06 (2006), ISO 1974 (1990), and ISO 2758 (2001).

\section{RESULTS AND DISCUSSION}

\section{Characterization of CCE Lye}

In the study of upgrading chemical pulp to dissolving pulp, CCE was the main method for removing hemicelluloses in the pulp, and its effects were confirmed by the experimental results. In the CCE process, the alkali and carbohydrates have very few chemical reactions, and the dissolution of hemicelluloses mainly depends on the swelling effect of the cellulose skeleton in a strong alkaline environment, so hemicelluloses can be regarded as physical dissolution during extraction. Thus, the characterization of CCE lye and isolated hemicelluloses (and possibly a small amount of degraded cellulose) was carried out to verify the value and recyclability of CCE lye and extend its application.

As shown in Table 2, the alkali concentration only decreased from $15.0 \%$ to $14.6 \%$ after the hemicelluloses isolation process, indicating that the reaction loss of alkali was very small, which is beneficial for the recovery and utilization of the alkali. The 
carbohydrates in the cold alkali extraction waste liquid were nearly $10.15 \%$ based on the raw material. The main sugar component was xylose, which accounted for $55.24 \%$ of the total carbohydrates, followed by mannose at $31.82 \%$. The rest of the carbohydrates were glucose $(8.04 \%)$ and small amounts of arabinose and galactose. For the hemicelluloses isolated from the CCE lye, the carbohydrate contents were also in the following decreasing order: xylose > mannose > glucose > galactose > arabinose. In addition, traces of glucuronic acid (GluA) and galacturonic acid (GlaA) were found in the isolated hemicelluloses, suggesting branched xylans (Peng et al. 2010). The total isolated hemicelluloses content was $10.75 \%$ based on the raw material, which was slightly higher than the total hemicelluloses dissolved in the CCE lye, implying that some lignin compound might also have been collected during ethanol extraction.

Table 2. Characteristics of CCE Lye before and after Hemicelluloses Isolation and Isolated Hemicelluloses

\begin{tabular}{|c|c|c|c|c|c|c|c|c|c|c|}
\hline \multicolumn{2}{|c|}{} & $\begin{array}{c}\text { NaOH } \\
(\%)\end{array}$ & $\begin{array}{c}\text { Glu } \\
(\%)\end{array}$ & $\begin{array}{c}\text { Xyl } \\
(\%)\end{array}$ & $\begin{array}{c}\text { Man } \\
(\%)\end{array}$ & $\begin{array}{c}\text { Ara } \\
(\%)\end{array}$ & $\begin{array}{c}\text { Gal } \\
(\%)\end{array}$ & $\begin{array}{c}\text { GluA } \\
(\%)\end{array}$ & $\begin{array}{c}\text { GalA } \\
(\%)\end{array}$ & $\begin{array}{c}\text { Total } \\
\text { Hemicelluloses } \\
(\%)\end{array}$ \\
\hline $\begin{array}{c}\text { CCE } \\
\text { Lye }\end{array}$ & Before & 15.0 & 8.04 & 55.24 & 31.82 & 3.15 & 1.75 & ND & ND & 10.15 \\
\hline $\begin{array}{c}\text { Isolated } \\
\text { Hemicelluloses }\end{array}$ & 14.6 & ND & ND & ND & ND & ND & ND & ND & ND \\
\hline
\end{tabular}

ND - too low to be detected; Glu - glucose; Xyl - xylose; Man - mannose; Ara - arabinose; Gal galactose. The monosaccharide percentages were calculated based on isolated hemicelluloses; the total hemicelluloses percentages were calculated based on raw material.

\section{Effect of CCE Lye on Deinking Process}

Cold caustic extraction lye was first used as an alkaline source to mix with deinking chemicals in the deinking process of waste newspapers (ONP). As shown in Fig. 1, by replacing alkaline solution with CCE lye (Fig. 1A vs. 1B), the strength properties of deinked ONP were improved. The tensile strength index increased by $31.0 \%$, the tear index increased by $5.3 \%$, and the burst index increased by $2.8 \%$.

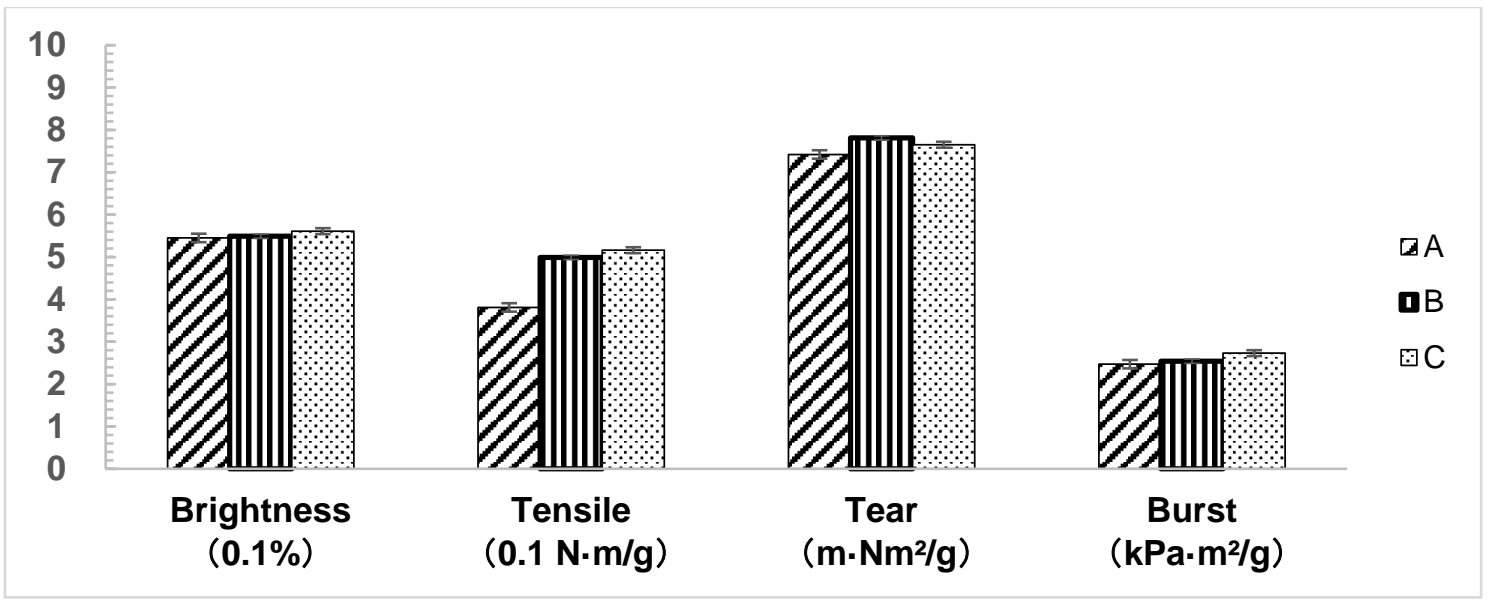

Fig. 1. Strength properties of deinked pulps: (A) $\mathrm{NaOH}$ as alkaline source, (B) CCE lye, and (C) CCE lye $+0.5 \%$ isolated hemicelluloses (based on pulp). Brightness and tensile index are presented multiplied by $1 / 10$ to be listed and compared with other data. 
To simulate the use of membrane treatment and other concentration technology to enhance the concentration of hemicelluloses in the CCE lye, $0.5 \%$ of hemicelluloses (based on ONP) was added in CCE lye used as an alkaline lye in the deinking process.

According to Tian et al. (2016), by applying nanofiltration to the CCE lye, the dissolved hemicelluloses can be concentrated from $25.3 \mathrm{~g} / \mathrm{L}$ to $59 \mathrm{~g} / \mathrm{L}$. By increasing the volumetric concentration factor through ultrafiltration, the concentration can be enhanced more (Sixta and Schild 2009). When using the CCE lye with hemicelluloses, the deinked pulp exhibited a similar brightness compared to the ordinary deinked pulp. The tensile index increased by $35.4 \%$, the tear index increased by $3.1 \%$, and the burst index increased by $10.5 \%$, compared to the ordinary deinked pulp, indicating the advantages of using CCE lye and concentrated CCE lye in the deinking process.

\section{Effect of CCE Lye on Bleaching Process}

In the bleaching process, hydrogen peroxide is often used in the last stage of multistage bleaching, and it is an indispensable part of total chlorine-free (TCF) bleaching. In the bleaching process, hydrogen peroxide not only can react with lignin, but it also decompose carbohydrates. Therefore, the strength of the chemical pulp after hydrogen peroxide bleaching is commonly reduced (Sjöström 1993). In addition, alkalinity plays a decisive role in the bleaching efficiency of hydrogen peroxide, as sufficient alkalinity can ensure that hydrogen peroxide plays an effective role in the ionization process. Based on the demand for alkalinity in hydrogen peroxide bleaching and the need to recover the loss of pulp strength after bleaching, CCE lye was applied to the hydrogen peroxide bleaching process to explore its utility in the bleaching sequence.

As shown in Fig. 2, when the unbleached pulp was treated in the hydrogen peroxide bleaching sequence using $\mathrm{CCE}$ lye instead of $\mathrm{NaOH}$, the brightness was not affected, and the tensile strength and burst strength of the bleached pulp were slightly improved $(11.4 \%$ and $1.8 \%$, respectively). However, the tearing strength was reduced by $19.5 \%$, indicating that the loss of fiber bonds by hydrogen peroxide bleaching may not be easily mitigated by the hemicelluloses in the CCE lye. When applying the concentrated CCE lye (Fig. 2C) to the hydrogen peroxide bleaching process, the strength properties of the bleached pulp were greatly improved by $2.9 \%$ to $18.0 \%$ (tensile by $17.9 \%$, tear by $2.9 \%$, and burst by $18.0 \%$ ).

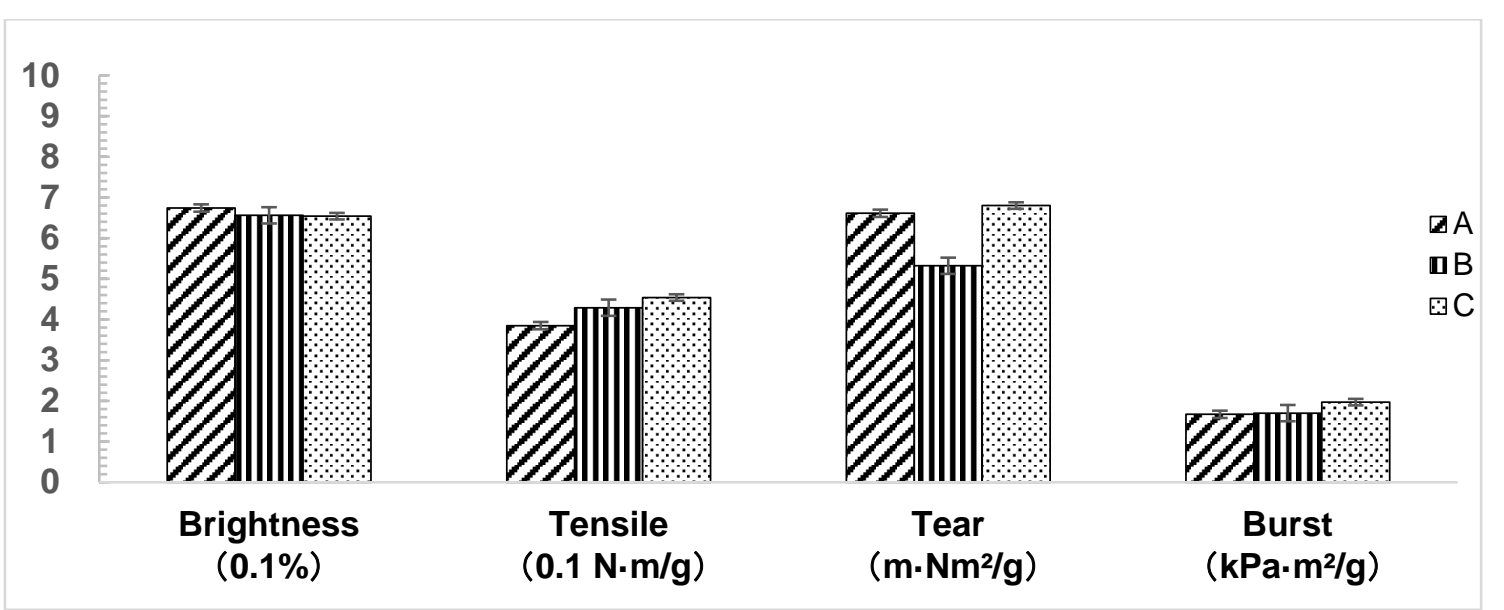

Fig. 2. Strength properties of $\mathrm{H}_{2} \mathrm{O}_{2}$ bleached pulps: (A) $\mathrm{NaOH}$ as alkaline source, (B) CCE lye, and $(C)$ CCE lye $+0.5 \%$ isolated hemicelluloses (based on pulp). Brightness and tensile index are presented multiplied by $1 / 10$ to be listed and compared with other data. 


\section{Effect of CCE Lye on Oxygen Delignification Process}

Oxygen bleaching is also a non-selective oxidation process, oxidizing lignin as well as carbohydrates such as cellulose, resulting in reduced pulp viscosity and strength. Generally, oxygen can only remove one third to one half of the residual lignin of unbleached pulp under alkaline conditions without causing serious loss of fiber strength. To improve the efficiency of oxygen delignification and inhibit the degradation of carbohydrates, the industry often uses acid, chlorine, and other pretreatments to remove transition metal ions or add cellulose-protective magnesium salt to makeup the pulp viscosity after oxygen delignification.

Experiments also show that the retention of hemicelluloses (especially polyxylose) or the addition of hemicelluloses to the pulp can effectively improve the strength properties of the paper (especially the tensile strength) while effectively reducing the energy consumption in the beating process (Dong et al. 2009). However, if the polyxylose is added separately, the cost will increase, which is not suitable for practical production. Therefore, CCE lye was applied to the oxygen delignification process in this study to test its efficiency. The properties of the oxygen-bleached pulps are compared in Fig. 3.

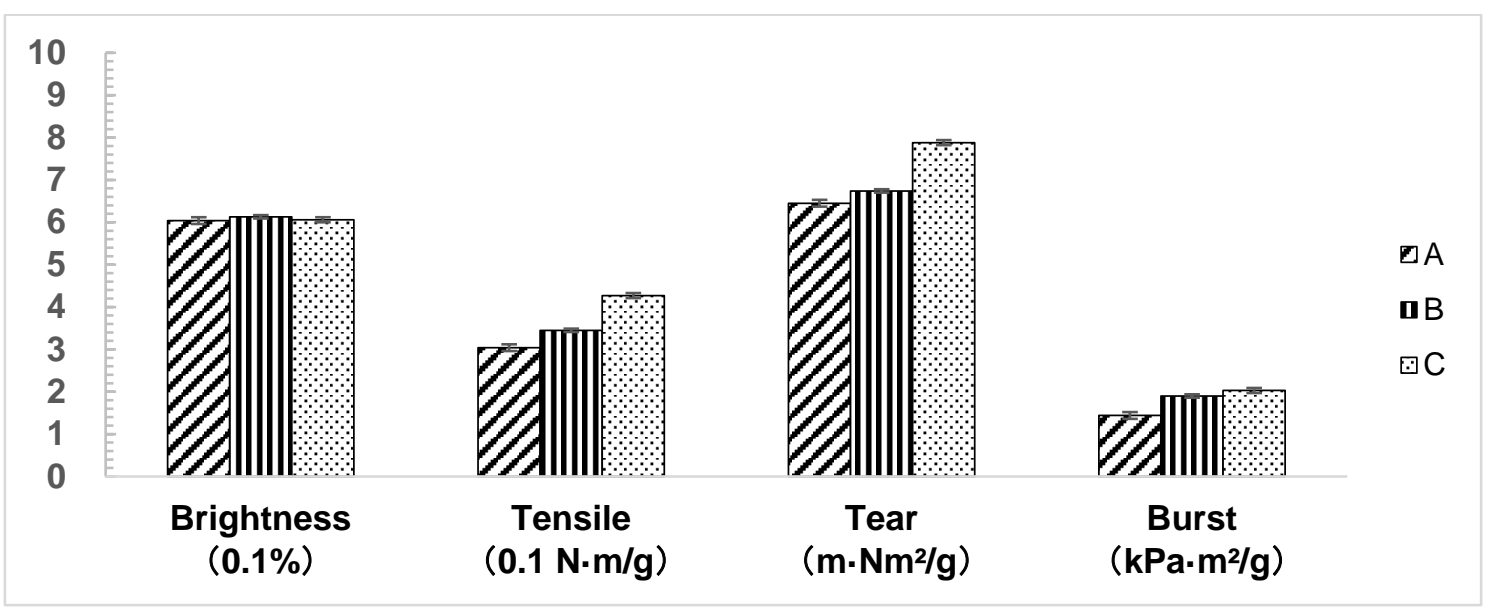

Fig. 3. Strength properties of oxygen-bleached pulps: (A) $\mathrm{NaOH}$ as alkaline source, (B) CCE lye, and (C) CCE lye $+0.5 \%$ isolated hemicelluloses (based on pulp). Brightness and tensile index are presented multiplied by $1 / 10$ to be listed and compared with other data.

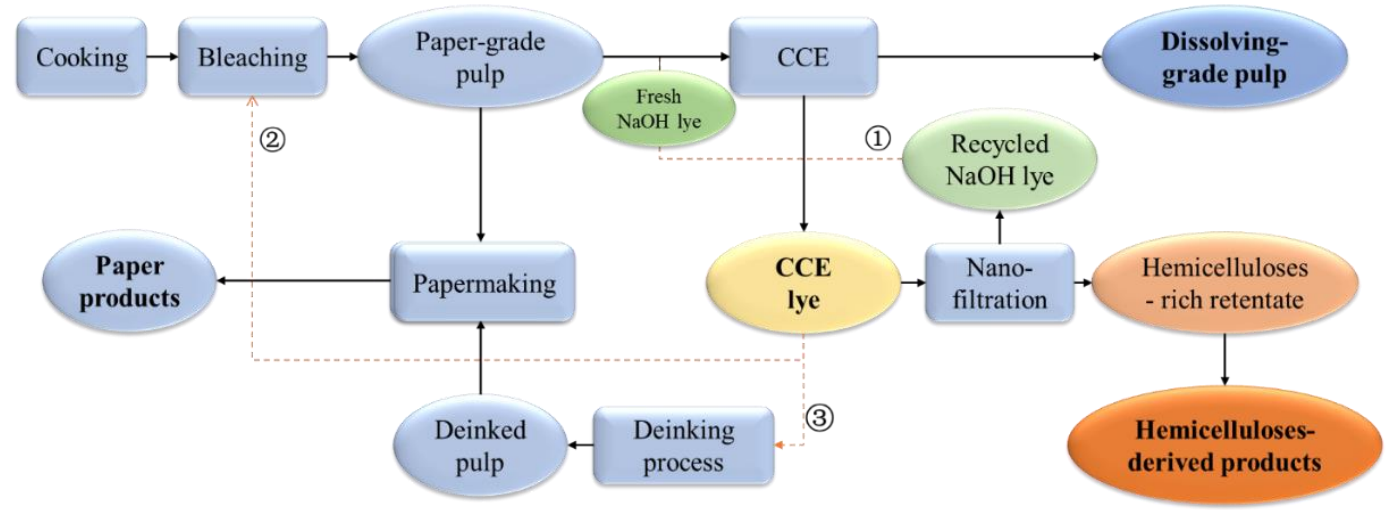

Fig. 4. The proposed biorefinery scheme integrating CCE to upgrade paper-grade pulp into dissolving pulp 
As shown in Fig. 3, when applying the CCE lye as an alkaline source in the oxygen bleaching, the strength properties of the bleached pulp were greatly improved, as tensile strength increased by $13.5 \%$, tear strength increased by $4.5 \%$, and burst strength increased by $31.9 \%$. These results indicated the efficiency of using CCE lye in oxygen bleaching to maintain the pulp strength. Meanwhile, the optical properties of the bleached pulp were maintained at a similar level.

In addition, using concentrated CCE lye (Fig. 3C) could further enhance the strength properties of the bleached pulp, as the tensile index increased by $40.5 \%$, tear index increased by $22.2 \%$, and burst index increased by $41.0 \%$, showing the marked effects of $\mathrm{CCE}$ lye on the oxygen bleaching process.

\section{CONCLUSIONS}

1. As a purification process of the dissolving pulp, cold caustic extraction (CCE) is a physical dissolution process. After isolation of hemicelluloses from the CCE lye, the alkali concentration of the CCE lye was reduced less than $1 \%$. The main carbohydrate components of the CCE lye were xylose and mannose, which accounted for $87.06 \%$ of the total carbohydrate, followed by $8.04 \%$ of glucose and small amounts of galactose and arabinose.

2. The CCE lye was tested in waste paper deinking, hydrogen peroxide bleaching, and oxygen delignification processes. Its efficiency in improving the strength performance of deinked pulp and bleached pulp was verified, as the tensile index increased by $11.4 \%$ to $31.0 \%$, the tear index increased by $4.5 \%$ to $5.3 \%$, and the burst index increased by $1.8 \%$ to $31.9 \%$, respectively.

3. Adding hemicelluloses to the CCE lye was studied to simulate the nano-filtration concentrated CCE lye. The results indicated that the concentrated CCE lye could further enhance the strength properties of deinked pulp and bleached pulp, as the tensile index increased by $17.9 \%$ to $40.5 \%$, the tear index increased by $2.9 \%$ to $22.2 \%$, and the burst index increased by $10.5 \%$ to $41.0 \%$, respectively.

4. The proposed integration scheme of the CCE process into the commercial pulp and papermaking process was presented (Fig. 4), showing good potential for the scale-up of CCE processes in practical production.

\section{ACKNOWLEDGMENTS}

This project was funded by the China Science and Technology Exchange Center (Grant No. 2016YFE0114700). 


\section{REFERENCES CITED}

Arnoul-Jarriault, B., Lachenal, D., Chirat, C., and Heux, L. (2014). "Upgrading softwood bleached kraft pulp to dissolving pulp by cold caustic treatment and acid-hot caustic treatment," Industrial Crops and Products 65, 565-571, DOI:

10.1016/j.indcrop.2014.09.051

Cao, B., Tschirner, U., and Ramaswamy, S. (1998). "Impact of pulp chemical composition on recycling," TAPPI Journal 81(12), 119-127.

Chen, J.-X. (1990). "Pulp bleaching," in: Pulping Principles and Engineering, Second Edition, Light Industry Press, Beijing, China, pp. 213-284.

China Paper Association (2020). "Review and outlook of dissolving pulp market in 2019," in: Almanac of China Paper Industry, China Light Industry Press, Beijing, China, 127-131.

Dong, C., Song, W., and Wang, Z. (2009). "Influence of adding hemicelluloses on the properties of recycled kraft pulp," Hunan Papermaking 2, 10-12. DOI:

10.3969/j.issn.1672-3066.2009.02.004

Gong, C., Shi, Y., Ni, J.-P., Yang, X.-B., Liu, Y.-Z., and Tian, C. (2017). "Integration of hemicellulose recovery and cold caustic extraction in upgrading a paper-grade bleached kraft pulp to a dissolving grade," Journal of Bioresources and Bioproducts 2(1), 20-23.

ISO 1974 (1990). "Paper — Determination of tearing resistance (Elmendorf method)," International Organization for Standardization, Geneva, Switzerland.

ISO 2470 (2016). "Paper, board and pulps - Measurement of diffuse blue reflectance factor," International Organization for Standardization, Geneva, Switzerland.

ISO 2758 (2001). "Paper — Determination of bursting strength," International Organization for Standardization, Geneva, Switzerland.

Oksanen, T., Buchert, J., and Viikari, L. (1997). "The role of hemicelluloses in the hornification of bleached kraft pulps," Holzforschung 51(4), 355-360. DOI: 10.1515/hfsg.1997.51.4.355

Peng, F., Ren, J.-L., Xu, F., Bian, J., Peng, P., and Sun, R.-C. (2010). 'Fractionation of alkali-solubilized hemicelluloses from delignified Populus gansuensis: Structure and properties," Journal of Agriculture and Food Chemistry 58(9), 5743-5750, DOI: $10.1021 / \mathrm{jf} 1003368$

Puls, J., Janzon, R., and Saake, B. (2006). "Comparative removal of hemicelluloses from paper pulps using nitren, cuen, $\mathrm{NaOH}$, and $\mathrm{KOH}$," Lenzinger Berichte 86, 63-70.

Sixta, H. (2006). "Pulp properties and applications," in: Handbook of Pulp, H. Sixta (ed.), Wiley-VCH Verlag GmbH \& Co. KGaA, pp. 1009-1067. DOI:

10.1002/9783527619887.ch11

Sixta, H., and Gabriele, S. (2009). “A new generation kraft process," Lenzinger Berichte 87, 26-37.

Sixta, H., Iakovlev, M., Testova, L., Roselli, A., Hummel, M., Borrega, M., van Heiningen, A., Froschauer, C., and Schottenberger, H. (2013). "Novel concepts of dissolving pulp production," Cellulose 20(4), 1547-1561. DOI: 10.1007/s10570-0139943-1

Sjöström, E. (1993). "Pulp bleaching," in: Wood Chemistry: Fundamentals and Applications, Second Edition, Academic Press, San Diego, CA, USA, pp. 173-187.

TAPPI T 494 om-06 (2006). "Tensile properties of paper and paperboard (using constant rate of elongation apparatus)," TAPPI Press, Atlanta, USA. 
Tian, C., Miao, Q., Zheng, L., and Ni, Y. (2016). "Upgrading paper-grade softwood kraft pulp to dissolving pulp by cold caustic extraction," Paper and Biomaterials 1(1), 3137. DOI: 10.12103/j.issn.2096-2355.2016.01.004

Zhang, M., Hubbe, M. A., Venditti, R. A., and Heitmann, J. A. (2002). "Can recycled kraft fibres benefit from chemical addition before they are first dried?," Appita Journal 55(2), 135-144.

Article submitted: December 9, 2020; Peer review completed: January 16, 2021; Revised version received and accepted: January 20, 2021; Published: January 22, 2021.

DOI: 10.15376/biores.16.1.1854-1862 